\title{
El papel de la autoevaluación en el proyecto interdisciplinar "formar docentes, formar personas". Una mirada desde la óptica de los estudiantes
}

\author{
Enrique Rivera García ${ }^{1 *}$, Carmen Trigueros Cervantes ${ }^{1}$, Alberto Moreno Doña ${ }^{2}$, \\ Eduardo de la Torre Navarro ${ }^{1}$ \\ ${ }^{1}$ Universidad de Granada (España) \\ ${ }^{2}$ Universidad Austral (Chile)
}

(Received September 25, 2011; Accepted February 20, 2012)

\begin{abstract}
RESUMEN: El proyecto "formar docentes, formar personas" es un intento de romper la inercia de la formación inicial del profesorado e inducir a nuestros estudiantes hacia una reflexión crítica desde tres ejes: interdisciplinariedad, autoevalución y participación. El trabajo interdisciplinar se construye desde tres asignaturas, buscando integrar a los docentes en un solo objetivo. Animamos a la participación democratizando el aula. Provocamos la responsabilidad del alumnado desde la confianza en su capacidad crítica y autocrítica para asumir el compromiso de su aprendizaje y, por ende, de la evaluación y calificación del mismo. La experiencia se presenta desde la doble mirada del docente y de los estudiantes, con el acento puesto en la evaluación. A la exposición de nuestros argumentos, hemos querido sumar las percepciones de nuestros alumnos, dejando al lector la puerta abierta para que pueda extraer sus propias conclusiones.
\end{abstract}

Palabras clave: interdisciplinariedad, formación inicial, educación física.

The role of self-assesment the interdisciplinary project «educating teachers, training people». A view from students' perspective assessing from self-assessment

\begin{abstract}
The project "educating teachers, educating people" is an attempt to go beyond the original training/education of teachers. This project tries to make our students think critically taking into account three different aspects: interdisciplinarity, self-assessment and participation. Interdisciplinarity is developed by three different subjects (will be later explained). We encourage participation by democratizing the classroom environment. We try to make students aware of their own responsibilities, and we do so by making them feel confident about their own critical abilities. Our intention is that they become used to assess themselves and in that way they become truly engaged in their learning process. The experience is presented from the point of view of the professor and also from the point of view of the student, stressing the importance of assessment process. Apart from our ideas/arguments, we wanted to include our students' perceptions, allowing the reader to arrive at their own conclusions.
\end{abstract}

Keywords: interdisciplinarity, pre-service teacher education, physical education. 


\section{INTRODUCCIÓN}

Si partimos de una concepción de la educación como espacio de participación, interacción y diálogo, es imprescindible escucharnos y escuchar la voz del alumnado. Escuchar y escucharnos, para abrir una puerta al análisis sobre la Formación Inicial de Profesores en la Universidad Española, especialmente ahora, en el que los procesos de convergencia con Europa cobran una gran relevancia en nuestra universidad. Pero realicemos una primera reflexión y analicemos en qué ha quedado la declaración de intenciones que se realiza desde el Bologna Working Group on Qualifications Frameworks (2005), cuando nos proponía una universidad para formar profesionales para el mercado de trabajo, ciudadanos activos que participen de las decisiones de una sociedad democrática, lograr un buen desarrollo personal y lograr mantener una base amplia de conocimiento puntero. Como plantea Huber (2008), al final del proceso las competencias se concentran en "conocimiento y comprensión, aplicación de conocimiento, evaluación y decisión, comunicación y destrezas de aprendizaje -ninguna palabra más sobre el desarrollo personal, valores, sentimientos, responsabilidad y la sociedad democrática-" (2008: 59). Otros autores como Martínez Bonafé (2004), orientan sus críticas al modelo de competencias por intentar homogeneizar la formación de docentes, denunciándolo como un nuevo discurso anclado en la lógica empresarial reinante en los sistemas educativos formales actuales. No le falta razón, además, es evidente que los cambios han sido formales: de organización y estructura del sistema. Sin entrar en los dos planos importantes: la mejora de las infraestructuras (Barbero, 2007; Devís y Peiró, 2007) y la formación de los docentes en las estrategias metodológicas y de evaluación que demanda el modelo.

La sensación que se percibe es como si la innovación pedagógica en la universidad se inicia con la convergencia europea. Nos olvidamos que muchos profesores llevamos tiempo trabajando a partir de lógicas pedagógicas y principios éticos, políticos y educativos para superar el modelo reproductor de conocimientos (tecnocrático). Tampoco pensemos que estamos ante un cambio radical; se trata de incorporar a la universidad "los principios pedagógicos de los movimientos de renovación escolar que se generaron en Europa y en España desde la segunda mitad del siglo XIX" (Masjuan, 2004: 73). Si analizamos brevemente estos principios pedagógicos y los traducimos al actual lenguaje del ECTS, podemos ver, como planteaba Shuell (1986) hace más de 30 años, que tenemos que centrarnos en facilitar la participación activa del estudiante en el proceso de enseñanza y aprendizaje propiciando que sea capaz de autorregular, construir, situar y socializar su aprendizaje. Como hemos podido ver, el andamiaje teórico no es nuevo, por lo que su aplicación generalizada a la enseñanza en la universidad podría haberse producido tiempo atrás.

¿Dónde hemos venido situando nuestro proyecto interdisciplinar "formar docentes, formar personas" (FDFP) a lo largo de esta última década? (Rivera y De la Torre, 2005). Dicho de forma sintética, hemos intentando convertir la formación inicial de maestros en un espacio educativo que no sólo forme profesionales con 
un conocimiento técnico adecuado, sino también con una visión de compromiso social, ético y pedagógico que contribuya, a partir de la educación, a transformar la sociedad consumista, neoliberal y discriminadora en la que estamos. Es una empresa difícil, pero no imposible, que nos debe movilizar para alcanzar la "eutopía" (Calvo, 2003) de una sociedad equitativa y en donde la justicia social sea el centro que permita convertir la democracia representativa presente en una verdadera democracia participativa.

Entendemos que precisamente lo olvidado de las primeras declaraciones del Bologna Working Group on Qualifications Frameworks es lo importante. Tenemos la sensación que estamos perdiendo la visión global por poner el acento en lo particular. Diseñamos competencias y subcompetencias, buscamos, al menos sobre el papel, la metodología más adecuada para su desarrollo y por último planteamos una estrategia de evaluación formativa que las haga visibles para poder terminar el proceso cuantificando y otorgando valor a todo aquello que hemos logrado visibilizar. ¿Dónde queda el desarrollo personal, los valores, el compromiso, los sentimientos, la responsabilidad, la implicación con lo colectivo,...? Que cada uno busque su propia respuesta.

\section{FORMAR DOCENTES, FORMAR PERSONAS. FORMAR PARA EDUCAR EN VALORES}

¿Qué es el proyecto FDFP? En la línea declarativa de Huber (2008) entendemos que la universidad debe tener un triple objetivo: preparar a los estudiantes para que puedan asumir su responsabilidad en el ámbito laboral desde la adquisición de los conocimientos y competencias necesarias, construir ciudadanos que participen activamente en una sociedad democrática y ayudarles a que aprendan a asumir sus responsabilidades personales. Tomando de referencia este triple objetivo, desde hace nueve años, venimos desarrollando en la Facultad de Educación donde trabajamos el proyecto interdisciplinar FDFP (De la Torre, Rivera y Trigueros, 2007; Rivera y De la Torre, 2005; Rivera, De la Torre. y Trigueros, 2009; Rivera, Trigueros, De la Torre y Moreno, 2010). Nuestros soportes se asientan por un lado en los postulados de la Pedagogía Crítica y en la idea de la democratización de los procesos formativos. Pretendemos la humanización de las personas, trabajando en la concienciación de los estudiantes hacia los nuevos problemas que se nos plantean desde una visión del mundo globalizado. Partimos de dos ideas claves. La educación no es neutral, siempre va a estar teñida de ideología que nutre el discurso e inunda la interacción entre docente y alumnado (Apple, 1986, 1987; Habermas, 1987,1991). Por esta razón, entendemos que la escuela es un espacio de interacción donde se construyen significados y no contextos de reproducción acríticos. (Apple, 1986; Carr, 1990; Freire, 1978, 1985; Giroux, 1990, 1992, 1993, 2001). Nuestra ideología pretende potenciar los valores universales, aquellos que la humanidad debe asumir como referente y que quedan perfectamente tipificados en el documento de la UNESCO: The Earth Charter Initiative (2003). En segundo lugar, entendemos que la educa- 
ción cobra sentido si es proyectada en la comunidad que la acoge, manteniendo como eutopía el logro de transformar la sociedad hacia modelos cada vez más democráticos, de participación, justicia e igualdad de oportunidades (MacLaren, 1989). Desde estas dos ideas ejes, se construyen cuatro pilares de trabajo: interdisciplinariedad, democratización del aula, metodología colaborativa identificada con los principios socio-constructivistas y autoevaluación. El último pilar: la autoevaluación es el que otorga sentido a todo el proyecto. Nuestra propuesta se construye desde un doble enfoque: la reflexión y la crítica; en un primer momento sobre sus propias acciones y en segundo lugar de las de sus compañeros, siempre con la idea puesta en la de-construcción de las teorías previas como punto de partida para la construcción del docente que deseamos ser. Analicemos en mayor profundidad los cuatro pilares del proyecto.

\section{Interdisciplinariedad}

El proyecto FDFP se nutre de tres materias: Educación Física y su Didáctica II, Didáctica del Juego Motor y la Iniciación Deportiva, y Danzas y Juegos Populares. Las dos primeras, al ser materias obligatorias, rompen tiempos y espacios y quedan integradas al cien por cien en el proyecto, compartiendo los mismos objetivos, contenidos, metodología y evaluación. En cuanto a la tercera materia, al ser optativa, no puede llegar al grado de fusión de las anteriores, pero sí comparte lo fundamental: los objetivos, especialmente los orientados al desarrollo de competencias personales y de interacción con los demás. Los contenidos son específicos, aunque comparte espacios de conferencias sobre temas transversales a las tres materias, metodología y evaluación. ¿Qué aporta al proceso de enseñanza y aprendizaje la interdisciplinariedad? Como docentes, nos permite trabajar desde una mirada global. Los tiempos de contacto con el alumnado aumentan, las interferencias entre materias desaparecen, la atención tutorial mejora en un cien por cien, especialmente en las dos primeras materias, ya que la ratio profesor-alumno se divide entre dos. La preparación de materiales se hace desde la colaboración, lo que reporta un ahorro en tiempo y energía. Los estudiantes descubren una visión de la Educación Física no atomizada. Se sienten partícipes de un proyecto, evitan la duplicidad de trabajos sobre temáticas muy similares. La evaluación es compartida, al igual que la calificación.

\section{Democratización del aula}

Entendida como participación, al tiempo que compromiso con la sociedad en la que se enmarca (Bernstein, 1988; Bilbeny, 1999), pero sin renunciar a la idea que expresa Touraine (1997) de una democracia hacia la liberación. Nuestra intención es hacer presente el concepto desde tres ejes: el estudiante debe ser libre para decidir el camino a recorrer. Sumarse implica compromiso a trabajar en colaboración, mantener una presencia y participación activa en los tiempos presenciales y dar respuesta a los mínimos marcados en la agenda del proyecto. 
En segundo lugar tendemos hacia un trabajo desde la horizontalidad, aunque somos conscientes de la dificultad de romper los roles preestablecidos. Para facilitar la igualdad rompemos la línea de poder dejando en sus manos los procesos de evaluación y calificación. De ellos será siempre la última palabra. Por último, nuestro tercer eje va a estar construido desde la tolerancia y la solidaridad. Como expresa Freire (1997: 44) "El aprendizaje de la tolerancia se realiza mediante el testimonio." Al mismo tiempo, entendemos la solidaridad basada en la igualdad, el respeto de la dignidad de todas las personas y la justicia (Habermas 1991).

\section{Metodología colaborativa identificada con los principios socio-constructi-} vistas

Conceptos como: globalización, interdisciplinariedad, aprendizaje significativo, contextualización etc... inundan a diario las aulas de las Facultades de Educación. La pregunta que se nos plantea es si las teorías constructivistas tan brillantemente explicadas (Bennett, 1979; Coll, 1987; Joyce y Weil, 1985; Montero, 1996; Ríos y Abascal, 1998; Trianes y Ríos, 1998) están pasando del discurso a la realidad del aula universitaria. ¿Cómo hemos aplicado los principios socioconstructivistas en nuestro proyecto? Básicamente dotando de significatividad a la propuesta desde un proyecto vinculado a la realidad escolar, cada grupo de estudiantes ha elaborado una propuesta de intervención para un aula de Primaria, desarrollando parte de la misma con los alumnos. En torno a este eje, se ha construido todo el andamiaje de los contenidos del proyecto FDFP: sustento teórico, propuestas prácticas simuladas y trabajo de investigación.

\section{Autoevaluación}

Asumir la responsabilidad del propio proceso de aprendizaje implica una toma de conciencia del mismo (Santos Guerra 2003). Si partimos de este punto y analizamos los principios que sustentan nuestro proyecto, nuestra única salida de cara a gestionar la evaluación es dejarla en manos de los protagonistas del mismo: los estudiantes. ¿Qué papel podemos asumir como docentes en esta parte del proceso? De facilitadores del mismo. Hacer reflexionar a los estudiantes sobre el significado social de la evaluación, identificar sus patologías y reconstruir el concepto (Fernández-Balboa, 2003; Santos Guerra 2003). Nuestra propuesta va en la línea marcada por Fernández-Balboa (2003: 2005). Desde el primer día de clase intentamos hacer ver a nuestros estudiantes que evaluar es tomar conciencia del proceso de aprendizaje, y que para ello deben buscar diferentes perspectivas.

En primer lugar la suya propia, desde un análisis crítico de su trabajo. Para este cometido les animamos a que utilicen dos herramientas fundamentales: el diario académico personal y el diario de aprendizajes. Desde el primero facilitamos la cristalización del proceso de aprendizaje vivido. Desde el segundo, queremos que la toma de conciencia se genere desde la "contabilidad" del trabajo realizado, tanto en los tiempos comunes de la presencialidad y no presencialidad. 
La segunda perspectiva que le ofrecemos es la del docente de referencia. Desde las tutorías grupales, se revisa el trabajo realizado a nivel grupal e individual aportando al estudiante nuestra opinión sobre el mismo. Además, desde la plataforma virtual, mantenemos un seguimiento constante sobre las propuestas que debe ir desarrollando.

La tercera perspectiva es la de sus propios compañeros. Se materializa básicamente desde la puesta en común de las propuestas, siempre finalizada con un debate de reflexión crítica donde se exponen los errores y posibles propuestas de mejora. Intentamos que se produzca la evaluación compartida entre iguales, animando a los estudiantes a buscar la opinión de un compañero respecto a un guión de lectura, una propuesta de intervención simulada para la práctica o sobre cualquier otro trabajo planteado.

La culminación del proceso de autoevaluación se produce al cerrar el semestre y se parte de la realización de la "guía de autoevaluación"; sería el equivalente al examen final de un enfoque tradicional. Es en este momento donde los alumnos tienen que revisar todo su proceso y hacer balance que, inevitablemente, por imposición del sistema, tiene que culminar con una auto-calificación. Aquí se pondría el punto y final a todo el proceso y les pedimos que esta auto-reflexión, la hagan pública con sus compañeros más directos de trabajo, aquellos con los que ha colaborado para poner en marcha la propuesta de intervención en el centro de Primaria (entre doce y catorce).

\section{ESCUCHANDO LA VOZ DE LOS ESTUDIANTES}

Hemos desgranado las bases del proyecto, los principios que lo sustentan y las estrategias que ponemos en acción para su desarrollo. Pero queremos hacer especial hincapié en la importancia que cobra la evaluación. Por esta razón entendemos la importancia de escuchar la voz de los participantes.

Para recabar la percepciones de nuestros estudiantes hemos utilizado los relatos escritos de la evaluación del proyecto realizada por ellos (Martínez Rodríguez, 2006). En el análisis de la información nos hemos apoyado en las propuestas de Strauss y Corbin (2002), Ruiz Olabuénaga, (2003) y Valles, (2003), utilizando el software para el análisis de datos cualitativos NVivo8 para profundizar en las narrativas y tener una visión global desde el análisis de lo particular.

¿Qué le pasaría a un estudiante si suprimiéramos de nuestras aulas universitarias el examen? Que brotaría en él la magia de la "motivación", vería su esfuerzo "compensado" por el reconocimiento que todos, profesores y compañeros, realizarían de su trabajo. Por esta razón, una gran parte de ellos son capaces de identificar entre el examen y procesos de evaluación donde él pasa a ser protagonista. Como uno de nuestros estudiante declaraba: "es una experiencia diferente al resto que buscan evaluar la exposición de unos conocimientos determinados en un día destinando para ello [lo] conocido como examen; permite que el alumno busque conocimientos más allá de su memorización para aprobar la asignatura."(Estudiante MN68563402). 
Constantemente, en nuestros discursos cotidianos en el aula de la Facultad de Ciencias de la Educación hacemos referencia a que el estudiante debe ser el protagonista, pero pocas, excesivamente pocas veces, somos capaces de brindarles la oportunidad, es: "algo difícil de ver hoy día en cualquier clase. Y la idea es buena, ya que solo uno conoce su grado de implicación, sus avances, su participación" (Estudiante HN44273024). Efectivamente, hay muchas competencias, especialmente aquellas que hacen referencia al saber ser y al saber estar en un contexto social, que su visibilidad depende fundamentalmente de su reflexión crítica; muy difícilmente seremos capaces de objetivarlas para su evaluación externa. Pero es más, para el resto de competencias su mirada siempre será más rica que la externa.

... hace que tengamos que hacer los propios alumnos la distribución del tiempo que le vamos a dedicar a la asignatura y que en la autoevaluación final seamos conscientes del trabajo que hemos realizado, y por tanto de la calificación que nos merecemos en base a conceptos como, la cantidad y calidad de aquellos que hemos aprendido, nuestro nivel de implicación o el tiempo que le hemos dedicado (Estudiante HN74731377).

Nos ha llamado la atención el miedo que para algunos estudiantes supone el hacer visible su evaluación (y calificación) en público, nosotros siempre hemos argumentado que un trabajo realizado en comunidad debe rendir cuentas a la comunidad, y desde esta posición siempre hemos defendido un proceso final público y abierto al debate y la crítica entre todos los participantes. En muchas ocasiones el estudiante prefiere la intimidad del confesionario (la tutoría), donde el sacerdote (el docente) hace visibles las faltas o las virtudes del pecador (el estudiante), y le impone la correspondiente penitencia en función de la gravedad del acto cometido. ¿Por qué el alumnado piensa siempre que tenemos la "verdad única y revelada" de nuestro lado? Pensamos que es el momento para cambiar estas creencias y comenzar a hacer visible que la verdad no es unívoca y menos en lo que se refiere a la educación.

La dificultad de "mirar" críticamente y de forma constructiva la mirada del “otro". ¿Cómo lograr que aparezca la empatía? Para poder ponernos en la piel de nuestro amigo, de nuestra amiga, del compañero o de la compañera con el que apenas cruzamos una palabra, en la piel de "ese" o de "esa" que no nos causa especial simpatía, del que siempre está dispuesto o dispuesta a acaparar la palabra cuando hay oportunidad de expresarnos en la clase, al tiempo que yo no soy capaz de levantar la mano para pedir una oportunidad y expresar lo que pienso. El proyecto tiene como uno de sus objetivos romper estas barreras del conocimiento superficial, ayudar a compactar el grupo y facilitar el conocimiento y el respeto entre todos los que integramos este microsistema en el que se transforma el aula. Aún reconociendo que la autoevaluación es "buena y nos hace crecer como personas y docentes, está bien pensado e ideado por una parte, pero por otra "hace agua" (Estudiante MN75430921); algunas personas no son capa- 
ces de respetar dos principios básicos: la honradez y la sinceridad de la persona" (Estudiante HN75165410).

El problema se genera cuando emerge la "competencia", cuando perdemos de vista nuestro aprendizaje y tenemos que valorar el del compañero o compañera ¿Es un problema generado desde el proyecto? Pensamos que no, la raíz del mal se encuentra en lo perverso del sistema al obligar a la jerarquización, clasificación y ordenación de los estudiantes, para poder seguir realizando el proceso de "selección natural" que organice y estratifique la sociedad. ¿Es necesaria la calificación? Creemos que debiera ser algo accesorio, carente de valor, salvo para determinar los mínimos que garanticen el poder mantenerte en los estudios. Existen otras formas de hacer explícito el nivel de competencia del estudiante, sin recurrir a una herramienta que contamina todo el proceso de enseñanza y aprendizaje, haciendo del medio el fin y objetivo principal.

Hablar de calificación implica que las críticas aparecen y la pérdida de la confianza en el "otro" se hace patente. ¿Dónde está el problema? Podríamos encontrarlo en la falta de empatía y el escaso respeto que nos merece la autocrítica de los demás. Por una parte decimos que nadie debiera juzgar nuestra actuación, al no tener suficientes criterios para ello, pero por otro lado pensamos que algunos "alumnos que no han trabajado tanto se han sobrevalorado a la hora de calificarse, debido a la forma de auto evaluación. La gente de la clase me han demostrado que salvo escasas excepciones, les falta dignidad y sinceridad" (Estudiante HA75116653).

El "hábitus" no hace al monje, pero condiciona enormemente las creencias del estudiante. Al sentirse acorralado, la solución que encuentran es volver a las viejas prácticas. Prefieren volver a la tutela del docente perdiendo su libertad individual, con tal de garantizar una hipotética justicia impartida desde la "verdad" del docente:

Otro aspecto que intentaría mejorar seria la autoevaluación debido a que hay mucha gente que no ha hecho nada e intenta aprovecharse de la confianza y el ambiente democrático que han conseguido establecer los profesores. Pienso que para evitar esto y que todo sea algo más justo, que los profesores puedan decidir quién va a examen y quien no y quien no vaya pues que entre en la autoevaluación tal y como la hemos llevado a cabo (Estudiante HN45603652).

Perdemos de vista algo fundamental, el proceso de autoevaluación es parte del aprendizaje, quizás el más determinante, especialmente llegada la hora de hacer explícitos los valores que dan sentido a los principios del proyecto. Como en todo aprendizaje está sometido a la posibilidad del error. La autocrítica se aprende sólo desde su práctica, especialmente en situaciones límites. El ejercicio público de la autoevaluación, y la culminación del mismo con la autocalificación, podríamos decir que es la máxima expresión de la capacidad de autocrítica del estudiante, es el momento de poner en juego la solidez de los valores que, se 
supone, debieran haber sido construidos a lo largo del proyecto.

Sienten que pierden la individualidad, pero no son conscientes del sentido de calificar como estrategia de posicionamiento respecto al grupo. Abandonar el "yo" y construir la identidad de pertenencia colectiva no es fácil, máxime si la constante a lo largo de todos sus años de escolarización es ver al "otro" como rival y no como compañero. Tener que calificarse, implica someter "a los alumnos a mucha presión y estoy seguro que en el $95 \%$ de los casos, la nota que los alumnos nos hemos puesto no es la que vosotros consideráis que nos merecemos" (Estudiante MN1463114). En esto se equivoca, nuestra percepción de las calificaciones se ajusta mucho a sus propuestas, salvo casos muy concretos, en los que expresamos nuestra disconformidad en la sesión pública, las distancias no suelen suponer más de 0,5 o a lo sumo 1 punto por encima o por debajo su calificación.

\section{CONCLUSIONES}

Después de una década, los cimientos del proyecto están consolidados. Nos sentimos con unos principios sólidos basados en el trabajo colaborativo y la asunción de unos valores universales como referente. En cuanto a los objetivos que nos marcamos cada día tenemos más claro que deben prevalecer aquellos que ayuden a desarrollar las competencias vinculadas con el ámbito personal y social. El conocimiento específico es necesario, pero solo si se proyecta en la realidad y cobra sentido, por lo que nos parece esencial la vinculación del aula universitaria con el aula de primaria. Metodológicamente tenemos la sensación de estar utilizando herramientas adecuadas que nos facilitan el aprendizaje desde la reflexión crítica (así entendemos la evaluación formativa). En cuanto a la evaluación mantenemos la apuesta por la auto-evaluación, a pesar de ser conscientes que al tener que desembocar finalmente en la auto-calificación la contamina y pone en riesgo. Pero como decimos en el epígrafe inicial, estas conclusiones son inacabadas y nuestro proyecto siempre está abierto, dispuesto a reconocer, desde la autocrítica y la mirada externa, otros senderos mejores para transitar.

\section{REFERENCIAS}

Apple, M. (1986). Ideología y curriculum. Madrid: Akal.

Apple, M. (1987). Educación y poder. Madrid: Paidós-MEC.

Barbero González, J. I. (2007). Incertidumbres en la formación de los maestros

y maestras de la EF escolar en el Marco de la Convergencia Europea. En L.

Martínez, y N. Bores (Coords.), La Formación del profesorado de Educación

Física en el marco de la convergencia europea. Valladolid: Universidad de Valladolid.

Bennett, N. (1979). Estilos de enseñanza y progreso de los alumnos. Madrid: Morata.

Bernstein, B. (1988). Clases, códigos y control. II. Hacia una teoría de las trans- 
misiones educativas. Madrid: Akal.

Bilbeny, N. (1999). Democracia para la diversidad. Barcelona: Ariel.

Bologna Working Group On Qualifications Frameworks (2005). A Framework for Qualifications of the European Higher Education Area. Extraído el 22 de abril de 2006 desde http://www.med.ub.es/ aprats/sae/docencia/creditosECTS.ppt.

Carr, W. (1990). Hacia una ciencia crítica de la educación. Barcelona: Alertes.

Coll, C. (1987). Psicología y currículum. Barcelona: Laia.

Calvo, C. (2003). Latinoamérica eutópica. En A. Elizalde, (Coord.), Las nuevas utopías de la diversidad. Lo deseable vuelve a ser posible. Santiago de Chile: Editorial Universidad Bolivariana, pp. 353-363.

De la Torre, E., Rivera, E., y Trigueros, C. (2007). Creencias y concepciones de la educación física en evolución: el caso de la formación del profesorado de educación física en la educación primaria. Tándem. Didáctica de la Educación Física, 23, 50-56.

Devís, J., y Peiró, C. (2007). La creación del espacio europeo de educación superior y la formación de los profesionales de la educación física y el deporte en España: iniciativas desde la Universidad de Valencia. En L. Martínez, y N. Bores (Coords.), La Formación del profesorado de Educación Física en el marco de la convergencia europea. Valladolid: Universidad de Valladolid.

Fernández-Balboa, J. M. (2003). La Auto-Evaluación (y la auto-calificación) como Formas de Promoción Democrática. En C. Moral, Materiales de Formación del Profesorado Universitario -Guía III- (pp. 97-106). Granada: UCUA (Unidad para la Calidad de las Universidades Andaluzas).

Fernández-Balboa, J. M. (2005). La autoevaluación como práctica promotora de la democracia y la dignidad. En A. Sicilia, y J. M. Fernández-Balboa (coord.), La otra cara de la enseñanza: La Educación Física desde una perspectiva crítica. (pp 127-158). Barcelona: INDE.

Freire, P. (1978) Pedagogía y Acción Liberadora. Madrid: Zero/Zyx.

Freire, P. (1985). Pedagogía del oprimido. Madrid: Siglo XXI.

Freire, P. (1997). La educación en la ciudad. México: Siglo XXI.

Giroux, H. (1990). Los profesores como intelectuales, hacia una pedagogía critica del aprendizaje. Madrid: Paidós-MEC.

Giroux, H. (1992). Alfabetización, poder y democracia. En H. Giroux, y R. Flecha (Eds.). Igualdad educativa y diferencia cultural,(9-26). Barcelona: El Roure.

Giroux, H. (1993). La escuela y la lucha por la ciudadanía. México: Siglo XXI. Giroux, H. (2001). Cultura, política y práctica educativa. Barcelona: Grao.

Habermas, J. (1987) Teoría y Praxis. Madrid: Tecnos.

Habermas, J. (1991). Tres modelos de democracia sobre el concepto de una política deliberativa. Debats, 39, 18-21.

Huber, G. L. (2008). Aprendizaje activo y metodologías educativas. Revista de Educación, número extraordinario, 2008, 59-81.

Joyce, B., y Weil, M. (1985) Modelos de enseñanza. Madrid: Anaya. 
MacLaren, P. (1989) La vida en las escuelas. México: Siglo XXI.

Masjuan, J. M. (2004) Convergencia europea, reformas universitarias, actitudes y prácticas de los estudiantes. Educar 33, 59-76

Martínez Bonafé, J. (2004). La formación del profesorado y el discurso de las competencias. Revista Interuniversitaria de Formación de Profesorado, 51, 127-143.

Martínez Rodríguez, J. B. (2006). Proyecto de investigación presentado para optar al cuerpo de Catedrático de Universidad. Material multicopiado no publicado.

Montero, L. (1996). Los estilos de enseñanza y las dimensiones de la acción didáctica. En C. Coll, J. Palacios y A. Marchesi (Coord), Desarrollo psicológico y educación II. (pp. 273-295). Madrid: Alianza Psicología.

Ríos, M., y Abascal, J. (1998). Modelo constructivista-contextual del aprendizaje: Vygotski y Bruner. En Trianes, M. V. y Gallardo, J. A. Psicología de la Educación y del Desarrollo. (pp. 401-421). Madrid: Pirámide.

Rivera, E. y De La Torre, E. (2005). Democratizar el aula universitaria: una propuesta alternativa de formación inicial universitaria desde la participación del alumnado. Investigación en la Escuela, 57, 85-95.

Rivera, E., De La Torre, E. y Trigueros, C. (2009). Formar docentes, formar personas: la formación inicial del profesorado desde una propuesta sociocrítica. Ciclo sobre Complejidad y Modelo Pedagógico. Extraído el 2 de julio de 2011 de http://www.tendencias21.net/ciclo/Un-centro-para-formacion-enla-complejidad-de-educadores-y-docentes-dara-continuidad-al-Ciclo_a68. html]

Rivera, E.; Trigueros, C.; De La Torre, E., y Moreno, A. (2010). Formar docentes, formar personas: una experiencia transdisciplinar para democratizar el aula universitaria. En L'Activitat del Docent: Intervenció, Innovació, Investigació. Barcelona: CiDd. II Congrés Internacional de Didáctiques, 1-8.

Ruiz Olabuénaga, J. I. (2003). Metodología de la investigación cualitativa. Bilbao: Universidad de Deusto.

Santos Guerra, M. A. (2003). Una flecha en la diana: la evaluación como aprendizaje. Madrid: Narcea.

Shuell,T. J. (1986). Cognitive conceptions of learning. Review of Educational Research, 56, 411-436.

Strauss, A., y Corbin, J. (2002). Bases de la investigación cualitativa. Técnicas y procedimientos para desarrollar la teoría fundamentada. Antioquia: Universidad de Antioquia.

Touraine, A. (1998). Igualdad y Diversidad. México: Fondo de Cultura Económica.

Trianes, M. V., y Ríos, M. (1998). Modelos cognitivos de aprendizaje escolar. En M. V. Trianes, y J. A. Gallardo, Psicología de la Educación y del Desarrollo. (pp. 375-399). Madrid: Pirámide.

Trigueros, C., Rivera, E., y De La Torre, E. (2006). Aprendizaje colaborativo en la formación de Maestros. Una experiencia práctica. Revista Tandem, 20, 45-55 
UNESCO. The earth Charter Initiative (2003). The Earth Charter. Extraído el 3 de julio de 2011) de http://www.earthcharterinaction.org/content/pages/ Read-the-Charter.html.

Valles, M. (2003). Técnicas Cualitativas de Investigación Social. Reflexión metodológica y práctica profesional. Madrid: Síntesis. 